\title{
Panorama general del consumo socialmente responsable en Bogotá
}

\author{
Overview of socially \\ responsible consume in Bogotá
}

\author{
Diana María López Celis (Colombia) \\ diana.lopez@utadeo.edu.co \\ Universidad Jorge Tadeo Lozano \\ Programa de Mercadeo
}

\begin{abstract}
Mónica Eugenia Peñalosa Otero (Colombia)
monica.penalosa@utadeo.edu.co

Universidad Jorge Tadeo Lozano

Programa de Mercadeo
\end{abstract}

\section{Resumen \\ El presente documento hace parte de la investigación que estamos desarrollando en conjunto con la Universidad de Murcia, titulada "Medición y caracterización del consumo socialmente responsable: análisis comparativo entre Colombia y España". \\ En este documento abordamos distintas teorías que se tienen sobre el consumo}

\begin{abstract}
Summary
This paper is part of a developing research in conjunction with University of Murcia, entitled "Measurement and characterization of socially responsible consume: comparative analysis between Colombia and Spain."

In this document, different theories about worldwide socially responsible consume (CnSR) are addressed, and
\end{abstract}

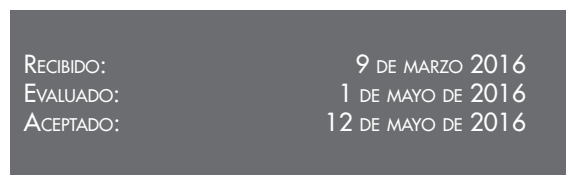

Para citar este artículo / to Cite this article López Celis, D. M. y Peñalosa Otero, M. E., (2016). Panorama general del consumo socialmente responsable en Bogotá, Bogotá: Editorial Politécnico Grancolombiano, Poliantea, 12(23), pág 35-51. 
socialmente responsable (CnSR) a nivel mundial, e indagamos con mayor profundidad acerca de lo que se conoce sobre este concepto en los consumidores de la ciudad de Bogotá, de estratos socioeconómicos 3 y 4 , dando a conocer los distintos vacíos de información que se tienen sobre el tema y mostrando cómo se está midiendo el CnSR en la actualidad por parte de estas personas de acuerdo con sus actitudes.

Palabras clave: consumo responsable, responsabilidad social, mercadeo, comunicación. what is known about this concept in 3 and 4 socioeconomic strata Bogotá city consumers is explored in depth, making known different information gaps on the subject and showing how the CnSR is currently being measured by these people according to their attitudes.

Keywords: responsible consume, social responsibility, marketing, communication. 


\section{Introducción}

El consumo socialmente responsable (CnSR), es un cambio de paradigma que se ha ido presentando alrededor del mundo. Se trata de una modificación en el pensamiento de los consumidores que los hace ser más conscientes acerca de lo que consumen y la manera en la que lo hacen (Dueñas, S., Perdomo, J. y Villa, L., 2014). En esta nueva forma de pensar se ve de manifiesto que los atributos de los productos han cambiado, y que otros factores como empaques biodegradables o procesos de elaboración amigables con el medio ambiente adquieren más relevancia a la hora de hacer la compra.

El CnSR es ampliamente conocido a nivel mundial, especialmente en Europa y en Estados Unidos, en donde se han podido identificar comportamientos, perfiles y rasgos característicos de los consumidores que siguen este modo de vida.

Recientes estudios llevados a cabo en diversos países señalan que las nuevas generaciones de consumidores, líderes en lo que deberá imperar como modelos de negocio en un futuro, conforman un segmento mucho más exigente y participativo en lo que se refiere a las actividades de responsabilidad social y ambiental de las empresas, hecho que efectivamente se ha encontrado relacionado con resultados positivos para las mismas (Mohr y Webb, 2005; Lecompte y Roberts, 2006; Cai y Aguilar, 2013; Cone Study, 2013).

Sin embargo, se ha evidenciado que hay vacíos de información que imposibilitan que se haga un perfil adecuado del consumidor en Colombia, y es por esto que este documento pretende abordar distintas 
teorías que se tienen sobre el consumo socialmente responsable (CnSR) a nivel mundial, e indagar el posible significado y las actitudes que tiene un consumidor socialmente responsable de la ciudad de Bogotá.

\section{Objetivo}

Profundizar en el conocimiento y actitud frente al consumo socialmente responsable en consumidores.

\section{Metodología de la investigación El estudio se realizó en dos fases}

En una primera fase se hizo una revisión de fuentes secundarias para la contextualización del consumo socialmente responsable y la responsabilidad social empresarial a nivel mundial, con lo cual se obtuvo un mayor conocimiento acerca del tema, tomando como referencia otras investigaciones y autores que han construido en torno al tema.

En una segunda fase, para obtener el pensamiento y actitud de los bogotanos, se realizó una investigación de alcance cualitativo, con un enfoque exploratorio, puesto que es la herramienta más eficaz para poder comprender este fenómeno desde la propia perspectiva de los consumidores.

\section{Técnica}

La técnica utilizada en este estudio fue la sesión de grupo.

\section{Target}

Consumidores bogotanos de estratos socioeconómicos 3 y 4 , con edades comprendidas entre 25 y 40 años.

\section{Muestra}

La sesión de grupo se realizó con 10 participantes de acuerdo con el target.

\section{Muestreo}

Para el estudio se tuvo en cuenta un muestro no probabilístico por conveniencia.

\section{Marco Teórico \\ El Consumo socialmente responsable}

El consumo socialmente responsable (CnSR) y la Responsabilidad social empresarial (RSE), constituyen dos caras de una misma moneda denominada consumo sostenible (Lorek y 
Spangenberg, 2014). El primero se ha definido como aquel realizado por un consumidor que "tiene en cuenta las consecuencias públicas de su consumo privado y quien intenta hacer uso de su poder de compra para lograr cambios sociales" (Webster, 1975, p. 188), mientras que el segundo se ha descrito como un "enfoque equilibrado de las organizaciones para hacer frente al tema económico, social y ambiental, de forma que busque beneficiar la comunicación con las personas y establecer lazos con la sociedad" (Leonard y McAdam, 2003, p. 27).

Según la revisión bibliográfica que, en relación con el estudio del consumo responsable, llevan a cabo Dueñas et al. (2014), el surgimiento de dicha tipología de consumo tiene su origen en los denominados consumidores verdes, en la investigación ecológica y del marketing que se dio en una etapa temprana de los años sesenta y en los movimientos medioambientales de la época (Hendarwan, 2002). El consumo verde es, según Elkington y Hailes (1989, p. 235), aquel que evita

... productos que ponen en riesgo la salud del consumidor o de otro; causan daño significativo al medioambiente durante la manufactura, su uso o desperdicio; consumen una cantidad desproporcionada de energía; causan un desperdicio innecesario; usan materiales derivados de especies o ambientes amenazados; así como aquellos que implican un maltrato innecesario de animales o que de manera adversa afectan a otros países.

Esta noción sobre el consumo verde se amplió rápidamente debido a que el aspecto ético y moral aparecía evidente en la toma de decisiones de los compradores, ampliándose el concepto al de consumidores éticos (Dueñas et al., 2014). En consecuencia, el concepto de consumo verde se refiere básicamente a aquellos consumidores que tienen preocupaciones medioambientales al adquirir sus productos o servicios, mientras que los denominados consumidores éticos son analizados teniendo en cuenta aspectos relativos a la moral en sus decisiones de compra o de adquisición de servicios (Dueñas et al., 2014).

Esta distinción es importante porque el proceso de toma de decisiones del consumidor ético presupone las preocupaciones medioambientales, y en este sentido es un concepto más amplio y complejo (Shaw y Shiu, 2002). Sin 
embargo, dentro de la literatura estos dos tipos de consumo están subsumidos a un ámbito más general donde se incluirían todos los aspectos sociales que tienen una influencia en las decisiones de consumo (Dueñas et al., 2014). El espectro amplio de asuntos sociales implícitos en el comportamiento y en la toma de decisiones de los consumidores es el que da lugar al concepto del consumo socialmente responsable (CnSR). El consumidor ahora no solo tiene en cuenta aspectos medioambientales y éticos, sino que en el acto de consumo involucra aspectos específicos como la responsabilidad social de las empresas, su contexto socioeconómico y cultural, o la información más allá de los propios bienes y servicios (Mohr et al., 2001; Ozcaglar-Toulouse, 2005; Webb et al., 2008).

El consumo socialmente responsable (CnSR), se origina como una respuesta a un mundo consumista, en donde el desperdicio de recursos y el consumo de bienes y servicios se hacen de manera desmesurada y descuidada. Para contrarrestar estos efectos se han firmado diversos acuerdos internacionales que permiten sobrellevar el impacto negativo sobre el ambiente. Entre los más destacados se aprecian el "Convenio marco sobre la diversidad biológica, la Declaración de Río sobre el medio ambiente y desarrollo y el Protocolo de Kioto de la Convención marco de las Naciones Unidas sobre el cambio climático" (Centro Andino de Integración, s.f.).

Esta clase de acuerdos internacionales, son los que han permitido que se modifique la forma en la que se producen bienes de consumo para que sean más amigables con el medio ambiente y responsables con la sociedad. Por su parte, las empresas han empezado a cambiar la manera en la que se producen los bienes que comercializan, adaptando una nueva cultura empresarial llamada Responsabilidad Social Empresarial (RSE), en donde, según Salas (2015), esta actividad consiste en:

Ofrecer elementos para garantizar el éxito de una empresa teniendo en cuenta consideraciones sociales y medioambientales en las actividades de la misma, para finalmente satisfacer la demanda de los consumidores y negociar al mismo tiempo las expectativas de los; trabajadores, proveedores y la comunidad de su entorno.

Gracias a esta nueva cultura, se ha ido produciendo un cambio en el consumo de las personas alrededor 
del mundo, haciéndolos más conscientes sobre todos los atributos de los productos, yendo más allá de un empaque atractivo o un precio razonable. Este cambio en el paradigma, podemos denominarlo consumo socialmente responsable (CnSR). Según Arenas (2006), citado por Oviedo y Paz (2010), este concepto trata de:

Aquellos comportamientos y decisiones de compra hechas por los consumidores que ponen en consideración los problemas ambientales, los recursos y una motivación guiada no solo por el deseo de satisfacer las necesidades personales, sino también por un interés ante las posibles consecuencias adversas y sus efectos consecuentes.

Respecto al CnSR se han hecho diversos estudios alrededor del mundo, principalmente en Estados Unidos y en Inglaterra, en donde se ha intentado operacionalizar diversas variables que tienen relación con actitudes socialmente responsables, como son el reciclaje, la reutilización de empaques, apoyo a negocios locales y demás, que permitirán realizar un posible perfil de consumidor. Siguiendo la línea europea, se evidencia que en España se han realizado diversos estudios sobre este concepto; en ellos se pudieron identificar distintos perfiles de consumidor de acuerdo con sus actitudes, sus principales necesidades psicológicas y sus rasgos fundamentales. Igualmente, se pudo evidenciar que es un concepto reconocido, pero por falta de disponibilidad de la información no se pone en práctica y los consumidores simplemente pierden el interés en tener un comportamiento más responsable frente al consumo.

Por su parte, en Latinoamérica se han realizado estudios en los que se ha clasificado al consumidor en siete perfiles que incluyen temas relacionados con el precio, la influencia que tiene internet en su vida social, al igual que como manejan sus ritmos de vida y la necesidad de pertenecer a un grupo de referencia (está relacionado con su estatus); a su vez, resalta el significado que tienen estos perfiles. Finalmente, permiten clasificar este consumidor psicológica y demográficamente.

Recientes estudios llevados a cabo en diversos países señalan que las nuevas generaciones de consumidores, líderes en lo que deberá imperar como modelos de negocio en un futuro, conforman un segmento mucho más exigente y participativo en lo que se refiere a las 
actividades de responsabilidad social y ambiental de las empresas, hecho que efectivamente se ha encontrado relacionado con resultados positivos para las mismas (Mohr y Webb, 2005; Lecompte y Roberts, 2006; Cai y Aguilar, 2013; Cone Study, 2013).

Según Cone Communications Social Impact Study (2013), las nuevas generaciones de consumidores ${ }^{1}$ tienen una imagen más positiva (93\%), creciendo en este sentido de los que afirmaban lo mismo en 2010 (un 85\%); confían más (un 91\%) y son más leales (89\%), a aquellas empresas que realizan acciones de apoyo social y medioambiental, así como también se muestran más dispuestos a comprar sus productos $y$ servicios (89\%), y a recomendarlas a otras personas (82\%).

Esta tendencia a considerar el compromiso social y ambiental de las empresas a la hora de tomar decisiones de compra y consumo parece indicar que estas nuevas generaciones tienen una mayor conciencia del poder de sus decisiones,

\footnotetext{
Se entiende por nuevas generaciones de consumidores a aquellas nacidas entre 1980 y el 2000, conocidos como Millennials en términos generacionales (Cone Study, 2013).
}

de la información de que disponen para ellas y de los beneficios o consecuencias que de ellas se derivan, algo de lo que quizás las generaciones previas no disponían.

\section{El marketing social}

El marketing social es nombrado por primera vez en 1971 por Gerald Zaltman y Philip Kotler en el artículo "Social marketing: An approach to planned social change"; en cuanto a la RSE, ya en el siglo XIX algunos empresarios industriales en Europa y en los EE.UU. se preocupaban por la vivienda, el bienestar y la caridad de sus empleados. Aunque la expresión surge entre los años 50-60 en EE.UU., no llega a desarrollarse en Europa hasta los 90, cuando la Comisión Europea, para involucrar a los empresarios en una estrategia de empleo que generase mayor cohesión social, utilizó el concepto.

Recientemente Kotler, Lee y Rothschild (2013), han descrito este concepto como "el proceso que aplica los principios y las técnicas de marketing para crear, comunicar y generar valor e influir así en comportamientos del público objetivo que benefician a la sociedad y a los propios consumidores". Estas 
campañas están encaminadas a promover aspectos específicos tales como problemas de salud, prevención de accidentes, cuestiones ambientales o participación ciudadana.

\section{La responsabilidad social empresarial en Colombia}

En la actualidad, no hay duda que dentro del contexto actual colombiano el tema de la responsabilidad social empresarial ha crecido significativamente en los últimos años. Para Asprilla (2010), las siguientes herramientas y principios demuestran el avance en el tema y los modelos más relevantes a la hora de implementar programas, basado en que una gestión socialmente responsable de las organizaciones deberá estar enmarcada dentro de los principios éticos y gerenciales mencionados a continuación:

1. El respeto a la dignidad de la persona humana, el cual es inherente a la naturaleza y destino trascendente del ser humano, igualmente le otorga derechos fundamentales irrenunciables y propios, de carácter personal, sociocultural y medioambiental, que las organizaciones deben reconocer y promover.

2. La responsabilidad legal, en donde las actuaciones de las organizaciones, no solo estarán enmarcadas dentro de la Constitución y las leyes que les apliquen, sino que se guiarán por la búsqueda proactiva de las normas aplicables, con el fin de asegurar el espíritu de las mismas.

3. La autorregulación ética, debido a que la gestión socialmente responsable implica una integración coherente de la misión y visión con el marco ético (valores y principios) que ha construido la organización.

4. La participación, partiendo del hecho de que los líderes han de promover que todo el personal se involucre en la gestión socialmente responsable, para que cada persona sea tenida en cuenta y que sus capacidades se pongan en función de los fines comunes de la organización y de la sociedad.

5. El enfoque adecuado de los procesos, debido a que los resultados deseados se alcanzan de manera más eficiente cuando las actividades y los recursos 
relacionados se gestionan como un proceso.

6. La solidaridad, entendida como la determinación firme y perseverante de trabajar por el beneficio mutuo, tal que las organizaciones y sus partes interesadas pueden alcanzar juntas aquello que aisladas no resulta viable.

7. El desarrollo humano integral como compromiso por preservar el patrimonio ambiental, cultural y social para las futuras generaciones, respetando la diversidad y promoviendo la reducción de las inequidades sociales, lo que implica que la organización integre en su estrategia los impactos económicos, sociales y medioambientales de su operación.

8. Por último, la mejora continua implica que las organizaciones guiarán con sentido ético el constante mejoramiento de sus procesos económicos, sociales y ambientales.
De acuerdo con la Confederación Colombiana de Cámaras de Comercio (Confecámaras), el modelo de implementación de prácticas de responsabilidad social empresarial en empresas colombianas es conocido como ComprometeRSE; el cual busca que las empresas definan sus objetivos e identifiquen cuáles serían los riesgos y oportunidades que se podrían presentar en el desarrollo estratégico del programa a implementar. De acuerdo con lo anterior, la organización debe realizar una investigación previa con el fin de conocer el estado actual de la RSE, saber y entender la fase en la cual se encuentran las prácticas dentro de cada organización.

El programa ComprometeRSE, luego de varios estudios, ha adelantado el modelo de implementación, el cual sirve como guía al consultor que acompaña a la empresa en la ejecución y sigue el ciclo P-H-V-A (Planear, Hacer, Verificar y Actuar) como se identifica en la figura No. 1. 
Figura 1: Modelo de implementación del programa ComprometeRSE

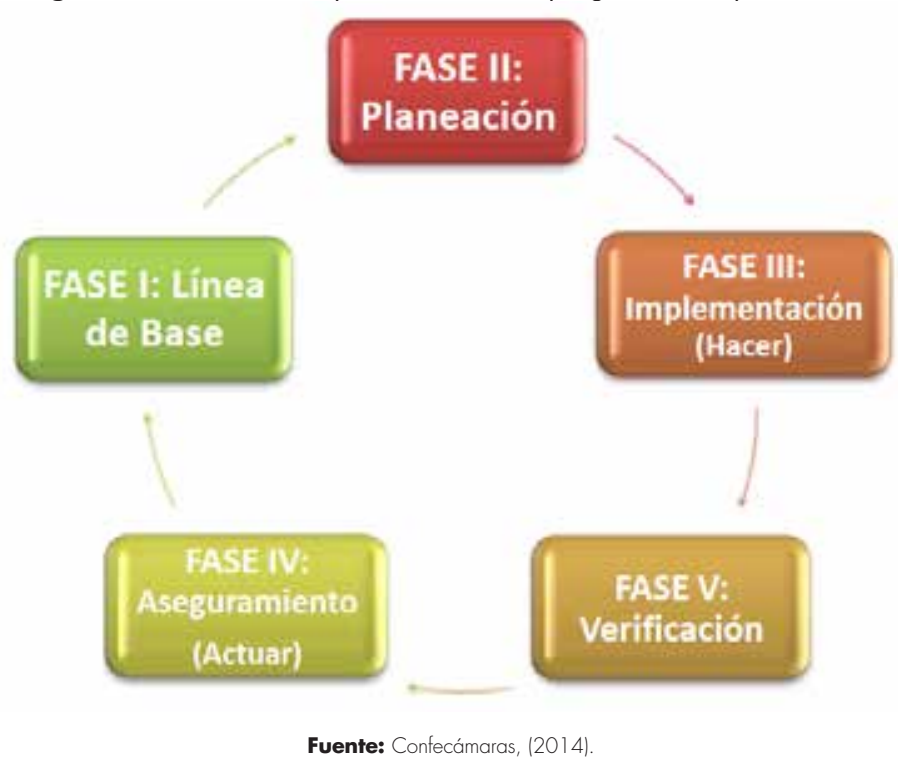

La Fase I o línea de base es el organización, además de un plan de punto de partida y debe establecer los cimientos en RSE de la empresa y el compromiso de la misma en el desarrollo e implementación de estrategias. Su objetivo es recopilar información relevante con el fin de elaborar un análisis interno y externo de la RSE, haciendo énfasis en las oportunidades y fortalezas que determinarán el enfoque de la organización en las siguientes fases.

La Fase II o planeación permite establecer objetivos y estrategias que respondan a las necesidades identificadas en la Fase I, tanto dentro de la empresa como de su entorno. Igualmente, debe incluir los lineamientos en RSE y las políticas de la comunicación interno y externo, un plan de acción, estratégico y operacional, así como la priorización y el diálogo con los stakeholders, conocidos también como grupos de interés.

La Fase III o de implementación materializa los objetivos de la organización en términos de RSE, y determina la eficacia de cada una de las actividades diseñadas en la fase anterior. Durante esta fase se deben ejecutar las estrategias de relacionamiento provenientes de los estudios de planeación. Es importante resaltar que la rigurosidad de la implementación depende el buen desarrollo del modelo. 
La Fase IV o de verificación reconoce que las labores de RSE, además de ser medidas, sean comunicadas. De la misma forma, hace visible ante los stakeholders los esfuerzos que realiza la organización en materia de sostenibilidad, siendo así una herramienta para promover la competitividad y divulgar la información. Esta fase, igualmente, busca realizar el seguimiento y la mediación de los procesos, políticas, objetivos y requisitos del modelo en desarrollo y el cumplimiento de los mismos. Finalmente, se debe verificar toda la información y garantizar que el lenguaje utilizado sea sencillo y apropiado para el sector al cual pertenece la empresa.

La Fase V o de aseguramiento vislumbra el momento de ciere del ciclo y tiene como objetivo recolectar lecciones aprendidas, aprendizaje y experiencias, convirtiéndose en el nuevo punto de partida para el siguiente proyecto. En esta fase se deben buscar tomar acciones para mejorar continuamente el desempeño.

\section{El consumidor colombiano}

Yepes (2012), hace una clasificación del perfíl de los consumidores responsables y sus principales características, entre las cuales se evidencia el cuestionamiento existente sobre los principios del sistema mercantil, el equilibrio entre las elecciones sostenibles y no sostenibles y finalmente el comportamiento y su variación en función de la situación y la circunstancia. De igual modo, es importante resaltar la importancia que tiene la dimensión identitaria y la reintroducción de valores al sistema económico.

Es por ello que se hace una distinción de las principales motivaciones que tienen las personas para practicar el consumo socialmente responsable desde la parte social, como por ejemplo la puesta en práctica de valores como la confianza y el respeto, la dimensión relacional desde el punto de vista del individuo, el conjunto social y el entorno; y desde una perspectiva ambiental, las diferentes visiones y percepciones que se tienen del concepto de medio ambiente y el cuestionamiento del sistema productivo a este respecto.

Por último, es importante analizar las reacciones frente a postulados de la economía neoliberal en donde se habla de utilitarismo, infinitud de la naturaleza y armonía de los intereses. 


\section{Resultados}

Frente al entendimiento del concepto de CnSR, los consumidores hacen una distinción en cuatro unidades principales de medida: medioambiente, necesidad/consumo, conciencia y cuidado de la salud. Es importante aclarar que el sentido del ahorro y de consumir justamente lo necesario, son variables constantes a la hora de definir el concepto. De igual modo, salta a la vista que la unidad de necesidad/consumo está ligada a los aspectos sociales y personales. Es allí en donde se empieza a pensar más en una sociedad colectiva que en una individualista; en donde se ve la necesidad de no malgastar los recursos por su escasez y por la necesidad de preservar el medio ambiente.

Al hablar de las prácticas más comunes de CnSR, las más recurrentes se dan en el ahorro de servicios públicos como el agua y la energía. El agua es el servicio que más se pretende cuidar; luego está el ahorro en luz, que tiene dos beneficios: el primero, permite ayudar a preservar el planeta, y el segundo, representa beneficios económicos. Otra práctica común es la del reciclaje; las personas se preocupan por saber en dónde poner sus desechos y cómo reciclar eficientemente, pero se comprobó que el sistema de recolección de basuras las junta en un mismo contenedor, imposibilitando que esta actividad se practique con más frecuencia.

En cuanto a la compra de productos de empresas que practican la responsabilidad social empresarial, se encontró que existe un gran desinterés por parte de los consumidores, ya que los atributos tradicionales que ofrecen las empresas fabricantes de los productos y que ofrecen servicios, son mucho más importantes que el hecho de que la empresa tenga buenas prácticas empresariales. De igual modo, es importante destacar que los consumidores sí son conscientes de que las empresas tienen este tipo de responsabilidad, pero esto no significa mucho para ellos, puesto que las campañas realizadas no resaltan esta práctica. A la larga son más importantes los productos de consumo y las marcas que lo atribuible a la sociedad o al medioambiente.

Con respecto a la percepción de los consumidores frente a las empresas que practican la responsabilidad social empresarial, se encontró que la gran mayoría de los participantes manifestaron que no creen en las campañas que se realizan, porque 
tienen la concepción de que el beneficio es mayor para la empresa que para la sociedad y en donde no existe una retribución justa. A pesar de que los consumidores conocen distintas campañas, no se sienten identificados con las mismas.

Con relación al impacto de la publicidad sobre el CnSr en los consumidores, se detectó que esta, en general, no motiva a los consumidores. Esto se debe principalmente a la falta de credibilidad que tienen las campañas de responsabilidad social empresarial en general. A pesar de que algunas campañas son más conmovedoras y directas, no logran un cambio actitudinal frente a los consumidores. La publicidad hecha por las empresas se encuentra mal enfocada, no se centra en mostrar las actividades de responsabilidad social que se están realizando, lo que hace imposible que los consumidores entiendan de una manera más precisa que las acciones de las empresas son reales y que buscan el beneficio de la sociedad.

\section{Conclusiones}

1. Para que los consumidores tengan en cuentan otros atributos diferentes a los tradicionales de un producto, es necesario desarrollar campañas de comunicación generen estímulos impactantes y sorpresivos; de esta manera es más probable que los consumidores procesen más rápido e internalicen el estímulo, debido a la novedad y naturaleza del mismo, logrando que las campañas tengan más impacto y que se empiece a generar un mayor aprendizaje con respecto al CnSR. En Colombia, Alpina es una empresa que siempre trabaja para generar confianza en sus alimentos, teniendo como horizonte la buena alimentación de las familias sin descartar en ninguno de sus procedimientos el gran compromiso que tiene con su comunidad, pues siempre está pensando en cómo trabajar por ella, el bienestar social y el mejoramiento y protección del medio ambiente. Igualmente, tiene muy claro su vínculo con el cliente, pues siempre lucha porque este esté bien informado y siempre cuida que se sienta bien y contento; después de todo, la marca es un gran representante de la empresa y además de vender una imagen, ofrecen una mayor conciencia. 
2. En cuanto a la credibilidad de las campañas de Responsabilidad Social Empresarial, es necesario que las organizaciones modifiquen su reputación, debido a que esta es una representación colectiva de las acciones pasadas de la organización y de sus propuestas futuras que describen cómo las audiencias clave interpretan las iniciativas de una entidad y evalúan su capacidad de entregar resultados. Actualmente, hay ciertos campos en donde hay participación pero no tan significativa como debería ser según la problemática que vive hoy día el país, pues aspectos como economía y comercio, empleo, medio ambiente y cultura y recreación son de gran importancia y tienen un alto potencial de acción. Por lo tanto, hay que seguir emprendiendo proyectos que apoyen esta causa, con el fin de generar una mejora en el desarrollo social y económico del país. Por ejemplo, la participación en construcción de viviendas impulsada con proyectos como el de la fundación TECHO de Home CenterSODIMAC, la cual fomenta la construcción de viviendas y me- jora las condiciones de estas, aun no se manifiesta de una manera más significativa comparándolo con la realidad y la necesidad de la población afectada, que en su mayoría son estratos 0 y 1 .

Por lo anterior, debe existir continuidad en las acciones de la empresa, en donde se dé un enfoque basado en la construcción de un nuevo valor de marca.

3. De igual modo, es necesario que se gestionen programas de refuerzo y que sean percibidos de manera positiva, para que de esta forma se logre un cambio perdurable y repetitivo en cuanto a las actitudes del consumidor bogotano. Es una realidad que al mostrarse beneficios tangibles sobre el consumo socialmente responsable, es más probable que los consumidores repliquen estas prácticas y que, a la vez, las promuevan en sus grupos de referencia por medio del voz a voz. Homecenter es una empresa en donde se desarrollan e implementan programas sociales de alto impacto que generan un valor agregado a los grupos de interés con los que interactúa la compañía (accionistas, empleados, clientes, proveedores, 
medio ambiente, gobierno y comunidad). Más allá del cumplimiento estricto de la ley, buscan garantizar un equilibrio entre lo filantrópico y lo económico, además de la sostenibilidad, vinculación y sensibilización de sus empleados y el reconocimiento por parte de sus clientes.

4. Para finalizar, es importante destacar que para darse un cambio de actitud debe generarse un trabajo en conjunto entre las empresas y los consumidores, teniendo en cuenta que este proceso parte de las organizaciones. Cualquier director de marketing y su equipo cumplen papeles esenciales en la educación, el empoderamiento y en la transformación de los hábitos de consumo de la sociedad.

\section{Referencias}

Asprilla, D. (2010). Proyecto de Guía Técnica Colombiana de Responsabilidad Social GTC-RS preparado para el Banco Interamericano de Desarrollo. Bogotá, Colombia.

Confecámaras - Confederación Colombiana de Cámaras de Comercio.
Recuperado de: http://www.confecamaras.org.co/

Dueñas, S., Perdomo, J. y Villa, L. (2014). El concepto de consumo socialmente responsable y su medición. Una revisión de la literatura. Estudios Gerenciales, 287-300.

Dinero (2015). Los mitos y verdades del consumidor colombiano. Recuperado de: http://www.dinero.com/pais/ articulo/los-mitos-verdades-del-consumidor-colombiano-estudio-kantarworldpanel/201574

García, L. y Betancourt, D. (2014). La racionalidad del consumidor, vista desde la conciencia social. Bogotá D.C: Pontificia Universidad Javeriana.

Gómez, I. (2010). Los estratos socieconómicos en Colombia. Recuperado de : http://igomeze.blogspot. com/2010/06/los-estratos-socioeconomicos-de.html

Lecompte, A. y Roberts, J. A. (2006). Developing a measure of socially responsible consumption in France. Marketing Management Journal, 16(2), 50-66.

Leonard, D. y McAdam, R. (2003). Corporate Social Responsability. Quality and Ethics, 27-32. 
Mohr, L. A., Webb, D. J. y Harris, K. Schiffman, L. y Kanuk, L. (2010). E. (2001). Do consumers expect com- Comportamiento del Consumidor. panies to be socially responsible? The México: Pearson. impact of corporate social responsibility on buyingbehavior. Journal of Consumer Affairs, 35(1), 45-72.

Oviedo y Paz (2010). El blog de Webster, F. (1975). Determining the Characteristics of the Socially Conscious Consumer. The Journal of ConOviedo \& Paz C.A. Recuperado de: https://oviedoypaz.wordpress. com/2010/10/07/\%C2\%BFque-esconsumo-socialmente-responsable/

Salas, C. (2009). El consumidor responsable en colombia. Bogotá D.C: Pontificia Universidad Javeriana. sumer Research, 2(3), 188-196.

Yepes, G. (2012). Consumo Responsable Fundamentos y práctica. Recuperado de: http://www.supersociedades.gov.co/inspeccion-vigilancia-y-control/gobierno-corporativo-y-rse/documentos/Documentos\%20RSE/Consumo_Responsable_Nov_2012\%20(7).pdf 